\title{
A Bioinformatic Analysis of Correlations between Polymeric Immunoglobulin Receptor (PIGR) and Liver Fibrosis Progression
}

\author{
Yuan Zhang $\mathbb{D}^{1},{ }^{1}$ Wenjun Lu $\mathbb{D},{ }^{2}$ Xiaorong Chen $\mathbb{D}^{1},{ }^{1}$ Yajuan Cao $\mathbb{D}^{\mathrm{D}},{ }^{3,4}$ and Zongguo Yang ${ }^{1}$ \\ ${ }^{1}$ Department of Integrative Medicine, Shanghai Public Health Clinical Center, Fudan University, Shanghai 201508, China \\ ${ }^{2}$ Department of Rheumatology and Immunology, The People's Hospital of Danyang, Affiliated Danyang Hospital of \\ Nantong University, Jiangsu 212300, China \\ ${ }^{3}$ Central Laboratory, Shanghai Pulmonary Hospital, School of Medicine, Tongji University School of Medicine, \\ Shanghai 200433, China \\ ${ }^{4}$ Clinical Translation Research Center, Shanghai Pulmonary Hospital, Tongji University School of Medicine, Shanghai 200433, China
}

Correspondence should be addressed to Yajuan Cao; yajuancao@tongji.edu.cn and Zongguo Yang; yangzongguo@shphc.org.cn

Received 14 January 2021; Revised 20 March 2021; Accepted 31 March 2021; Published 12 April 2021

Academic Editor: Udayan Apte

Copyright (C) 2021 Yuan Zhang et al. This is an open access article distributed under the Creative Commons Attribution License, which permits unrestricted use, distribution, and reproduction in any medium, provided the original work is properly cited.

\begin{abstract}
Objective. This study is aimed at investigating the enriched functions of polymeric immunoglobulin receptor (PIGR) and its correlations with liver fibrosis stage. Methods. PIGR mRNA expression in normal liver, liver fibrosis, hepatic stellate cells (HSCs), and hepatitis virus infection samples was calculated in Gene Expression Omnibus (GEO) and Oncomine databases. Enrichment analysis of PIGR-related genes was conducted in Metascape and Gene Set Enrichment Analysis (GSEA). Logistic model and ROC curve were performed to evaluate the correlations between pIgR and liver fibrosis. Results. PIGR mRNA was upregulated in advanced liver fibrosis, cirrhosis compared to normal liver (all $p<0.05$ ). PIGR mRNA was also overexpressed in activated HSCs compared to senescent HSCs, liver stem/progenitor cells, and reverted HSCs (all $p<0.05$ ). Enrichment analysis revealed that PIGR-related genes involved in the defense response to virus and interferon (IFN) signaling. In GEO series, PIGR mRNA was also upregulated by hepatitis virus $\mathrm{B}, \mathrm{C}, \mathrm{D}$, and $\mathrm{E}$ infection (all $p<0.05$ ). After adjusting age and gender, multivariate logistic regression models revealed that high PIGR in the liver was a risk factor for liver fibrosis $(\mathrm{OR}=82.2, p<$ 0.001). The area under curve (AUC), positive predictive value (PPV), negative predictive value (NPV), sensitivity, and specificity of PIGR for liver fibrosis stage $>2$ were $0.84,0.86,0.7,0.61$, and 0.90 . Conclusion. PIGR was correlated with liver fibrosis and might involve in hepatitis virus infection and HSC transdifferentiation.
\end{abstract}

\section{Introduction}

The polymeric immunoglobulin receptor (PIGR) is exclusively originated from intestinal epithelial cells. It captured and transcytosed dimeric $\operatorname{Ig} \mathrm{A}(\operatorname{dIgA})$ from lamina propria to intestinal lumen across epithelial cells and participated in mucosal immune system $[1,2]$. Previous evidences revealed that proinflammatory cytokines released by innate and adaptive immune cells including interferon, tumor necrosis factor (TNF), interleukins, and lymphotoxin could stimulate the expression of PIGR [1-9]. Except for exerting receptor function, the extracellular portion of PIGR is also responsible for intracellular neutralization of some viruses $[10,11]$.
Recently, PIGR has been proved to be involved in the human tumorigenesis and malignancies [9, 12-14]. As a vital inflammatory mediator [1], PIGR played an important role in hepatitis B (HBV) infection, chronic liver inflammation, tumor growth, recurrence, and metastatic progression in liver cancer and pancreatic ductal adenocarcinoma $[9,12$, 14]. Mechanistically, PIGR had a cross talk of transforming growth factor- $\beta$ with inflammatory mediators like tumor necrosis factor- $\alpha$, interferon- $\gamma$, and interleukin- 4 , resulting in the induction of epithelial-mesenchymal transition (EMT) [13]. Moreover, PIGR upregulation increased the nuclear translocation of $S m a d 2 / 3$, leading to the activation of Smad pathway [12]. Rac1/CDC42-MEK/ERK cascade11 
was also proved to be potential mechanism of PIGR-related cancer malignancy [14]. We previously found that PIGR involved in the activation of ribosome pathway and accounted for liver cancer recurrence [15]. Upregulated in alcoholic liver disease, PIGR isoform X1 has been identified as a predictor for liver fibrosis [16]. Additionally, PIGR was overexpressed in cirrhosis compared to nonalcoholic fatty liver disease. A global correlation map of clinical and proteomic data strongly associated PIGR with liver cirrhosis [17].

In this study, we aimed to investigate the expression of PIGR according to liver fibrosis, status of hepatic stellate cells (HSCs), and hepatitis virus infection. By identifying the associations between PIGR and liver fibrosis progression, we hope to offer novel insights into the mechanisms of liver fibrosis, even in hepatocarcinogenesis.

\section{Materials and Methods}

2.1. Gene Expression Omnibus (GEO). GEO database [18, 19] was searched with heading terms including "liver fibrosis," "cirrhosis," "hepatitis," "hepatitis virus," "hepatitis A (HAV)," "hepatitis B," "hepatitis C (HCV)," "hepatitis D (HDV)," and "hepatitis E (HEV)." All the series with expression profiling by array were included. No sample type and organism type restriction. Platforms and samples of GEO series were downloaded from https://www.ncbi.nlm.nih .gov/geo/.Raw.CEL files of the microarray from each GEO dataset were normalized by quantile method of Robust Multichip Analysis (RMA) from R affy package [20]. Gene expression comparison was calculated by Limma package in $\mathrm{R}$ program version 4.0 [21]. The details of GEO series included in this analysis were summarized as Supplementary Table S1.

2.2. Oncomine. The Oncomine is a cancer microarray database and web-based integrated data mining platform aimed at facilitating discovery from genome-wide expression analyses [22]. With more than 700 independent datasets and a collection of over 18000 microarray experiments, the Oncomine platform provides solutions that can compute gene expression signatures, clusters, and gene-set modules, automatically extracting biological insights from the data [22, 23]. Studies compared PIGR between normal liver and cirrhosis samples were selected without threshold, fold change, and gene rank restriction in Oncomine database (https://www.oncomine .org/).

2.3. The Human Protein Atlas (HPA). The HPA program mapped all the human proteins in cells, tissues, and organs using an integration of various omics technologies, including antibody-based imaging, mass spectrometry-based proteomics, transcriptomics, and systems biology [24]. PIGR protein in tissue atlas and PIGR mRNA in cell type atlas of liver were obtained from HPA database.

2.4. DisGeNET. DisGeNET is a discovery platform containing one of the largest publicly available collections of genes associated to human diseases [25-27]. Associated diseases of PIGR were searched in DisGeNET version 6.0 (https:// www.disgenet.org/). Summaries and evidences of gene- disease associations (GDA) including semantic type, scores of GDA, and numbers of publications in PubMed were all obtained.

2.5. Enrichment Analysis. Using Similar Genes Detection function in Gene Expression Profiling Interactive Analysis (GEPIA) database, top 100 similar genes of PIGR with similar expression pattern in LIHC normal liver dataset were identified [28]. Protein-Protein Interaction analysis (PPI) for PIGR was investigated by STRING version 11.0 (https:// string-db.org/) and STITCH version 5.0 (http://stitch.embl .de/) databases. All these interacted genes and similar genes of PIGR in GEPIA, STRING, and STITCH were included in Metascape for enrichment analysis [29]. Top ten Kyoto Encyclopedia of Genes and Genomes (KEGG) pathway, Gene ontology (GO) biological process, and Reactome enrichment analysis were also investigated in Molecular Signatures Database in Gene Set Enrichment Analysis (GSEA, http:// software.broadinstitute.org/gsea/index.jsp) version 4.1 with a false discovery rate $(\mathrm{FDR}) p$ value $<0.05[30,31]$.

2.6. Statistical Analysis. Differences of PIGR expression levels between the individual groups were analyzed using Student's $t$ test or Mann-Whitney test based on variable types by GraphPad Prism 8 (GraphPad Software, San Diego, CA, USA). Parameters associated with the liver fibrosis stage were assessed by univariate and multivariate logistic regression by Stata software version 16.0 (Stata Corp LLC, Texas, USA). Results were reported as odds ratios (OR) with 95\% confidence intervals (CI). OptimalCutpoints package [32] in $\mathrm{R}$ program was used to perform ROC analysis to evaluate predictive values of potential factors for the liver fibrosis stage. A two-tailed $p<0.05$ were considered significant for all tests.

\section{Results}

3.1. PIGR Expression in Different Stages of Liver Pathology. In Oncomine database, Wurmbach Liver and Mas Liver datasets reported PIGR comparison between cirrhosis and normal liver. Microarray experiments of 13 cirrhosis samples and 10 normal liver samples in Wurmbach Liver dataset were addressed in Human Genome U133 Plus 2.0 Array platform [33], and Transcriptome levels of PIGR in 19 normal liver samples and 58 cirrhosis samples in Mas Liver dataset were examined in Human Genome U133A 2.0 Array platform [34]. As shown in Figure 1(a), PIGR mRNA was significantly upregulated in cirrhosis tissues than that in normal liver in Wurmbach Liver dataset $(p<0.0001$, Figure 1(a)). Conversely, PIGR mRNA was downregulated in cirrhosis samples than that in normal liver in Mas Liver dataset $(p<0.0001$, Figure 1(b)). GEO series were also selected for investigating PIGR expression between cirrhotic and normal livers. The details of the included GEO series were summarized in Supplementary Table S1. As shown in Figure 1(c), PIGR mRNA was significantly overexpressed in cirrhotic samples than that in normal livers in GSE7741 and GSE25097 $(p<0.01$ and $p<0.0001$, respectively, Figure $1(\mathrm{c}))$. On the contrary, PIGR was downregulated in cirrhosis compared to that in healthy individuals in 


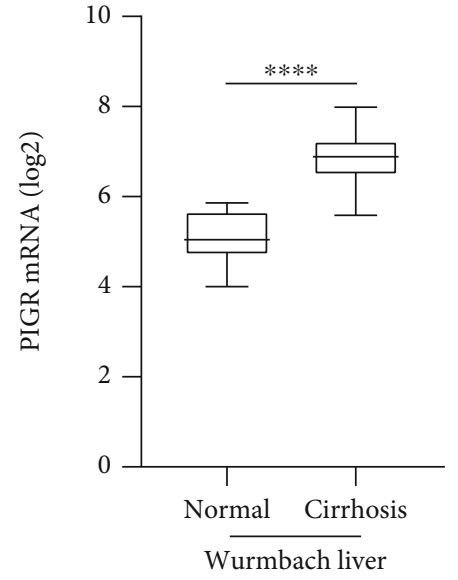

(a)

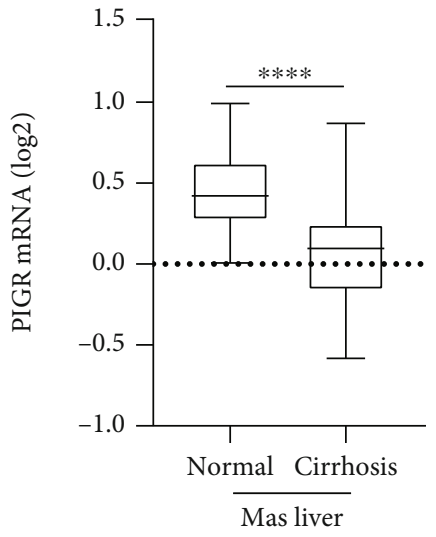

(b)

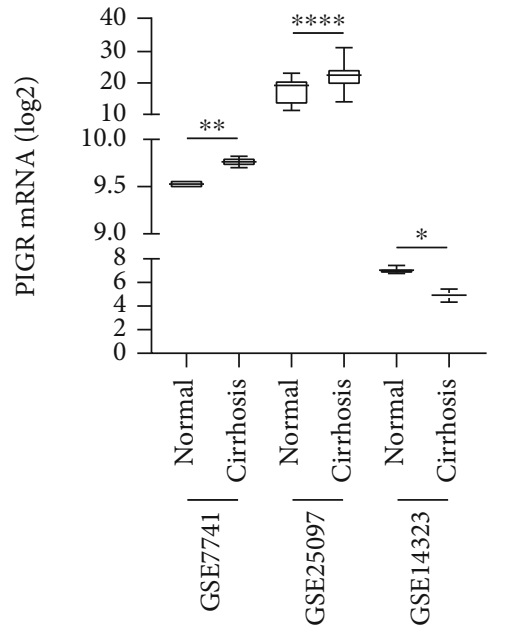

(c)

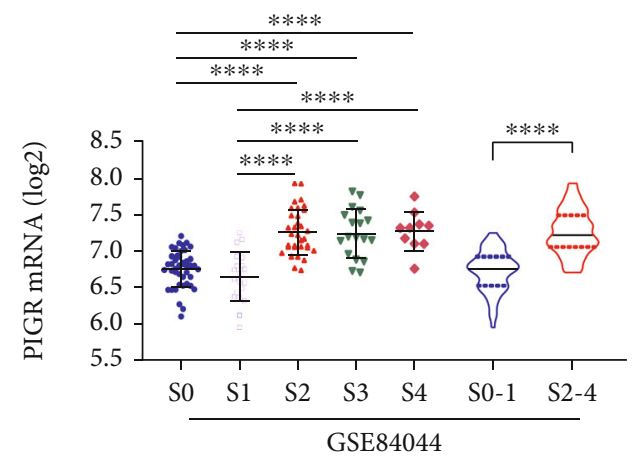

(d)

FIGURE 1: PIGR mRNA expression between normal and cirrhosis samples. PIGR was upregulated in cirrhosis than that in normal samples in Wurmbach Liver $(p<0.0001$, a) and downregulated in cirrhosis in Mas Liver $(p<0.0001, \mathrm{~b})$. In GEO series, PIGR was overexpressed in cirrhosis samples compared to normal livers in GSE7741 and GSE25097 and downregulated in cirrhotic samples in GSE14323 (c). Compared to liver fibrosis stages 0-1, PIGR elevated in patients with liver fibrosis stages 2-4 $(p<0.0001, \mathrm{~d})$.

GSE14323 ( $p<0.05$, Figure 1(c)). In GSE84044, liver samples of 124 chronic hepatitis $\mathrm{B}(\mathrm{CHB})$ patients were examined [35]. As shown in Figure 1(d), PIGR mRNA was significantly overexpressed in $\mathrm{CHB}$ patients with fibrosis stage $\geq 2$ compared to that in patients with fibrosis stage $<2$ (all $p<0.001$, Figure 1(d)).

3.2. PIGR Expression in Liver Cells and HSCs. PIGR protein in liver tissues was obtained from HPA database. As shown in Figure 2(a), PIGR was not detected in all eight cholangiocytes, while it was medium/low staining in six of eight hepatocytes (Figure 2(a)). PIGR RNA expression in the single cell type clusters identified in liver tissue was summarized in Figure 2(b). PIGR mainly expressed in cholangiocytes, followed by hepatocytes (Figure 2(b)).

Considered the pivotal roles of HSCs in the development of liver fibrosis, PIGR mRNA expression levels were identified in HSCs in GEO series including GSE11954 [36], GSE49995 [37], and GSE68001 [38]. In GSE11954, two separate preparation of activated HSCs were treated with DNA damaging agent to induce senescence or vehicle to remain growing [36]. Compared to senescent HSCs, PIGR in growing HSCs was significantly overexpressed $(p<0.01$, Figure 2(c)). In GSE49995, PIGR expression in 7 samples of adult-derived human liver stem/progenitor cells (ADHLSCs) and 7 samples of HSCs were measured [37]. PIGR mRNA was significantly upregulated in HSCs compared to that in ADHLSCs $(p<0.001$, Figure 2(c)). In GSE68001, HSCs were isolated from healthy liver and culture-activated as aHSCs, and aHSCs were reverted by reverting medium and displayed as a quiescent-like phenotype (rHSCs) [38]. Compared to rHSCs, PIGR was significantly upregulated in aHSCs $(p<0.001$, Figure 2(c)).

3.3. Enrichment of Similar/Interactive Genes of pIgR. Top 100 similar genes of PIGR in LIHC normal liver dataset were identified in GEPIA database (Figure 3(a)). PPI of PIGR was evaluated in STRING and STITCH databases (Figures 3(b) and 3(c), respectively). Enrichment analysis of similar/interacted genes of PIGR was calculated in 


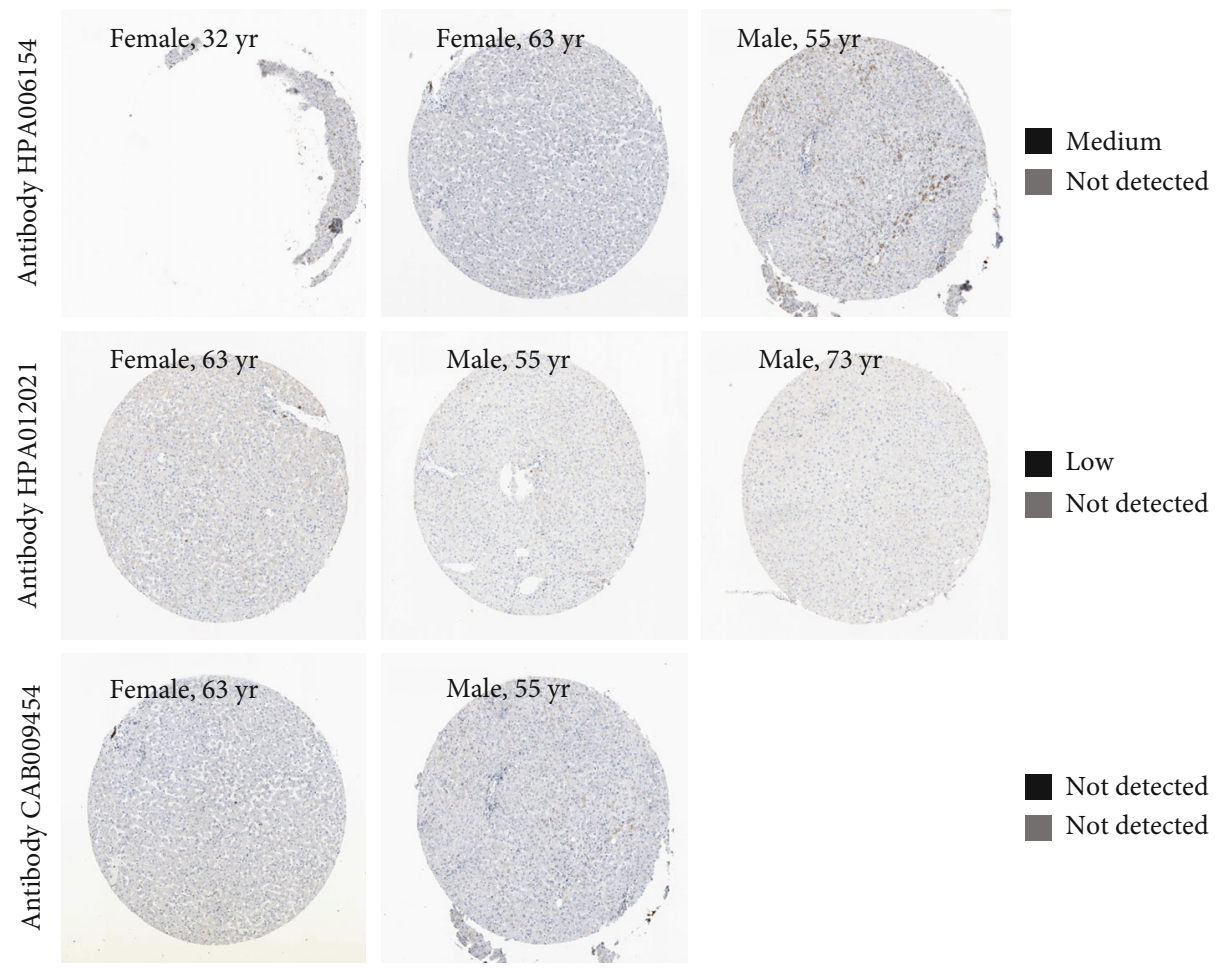

Hepatocytes

Cholangiocytes

(a)

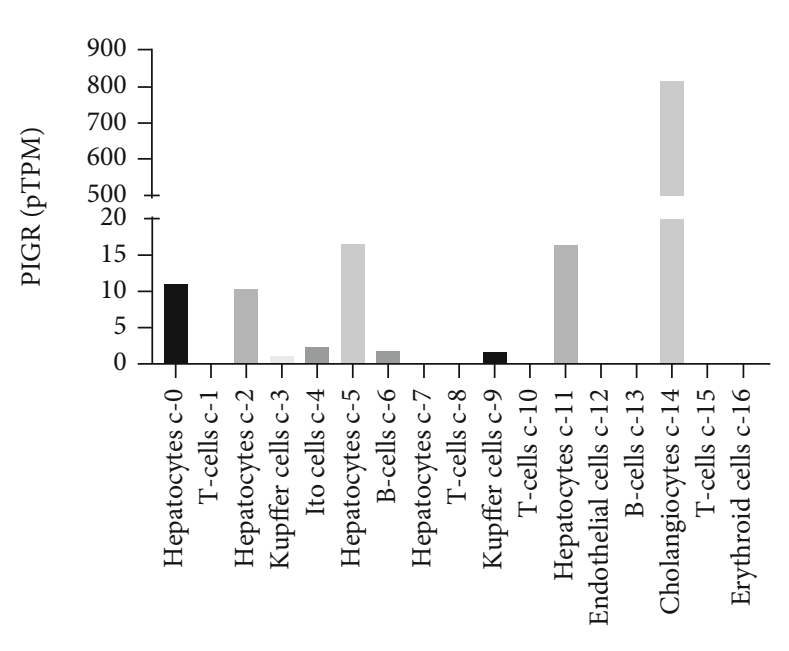

(b)

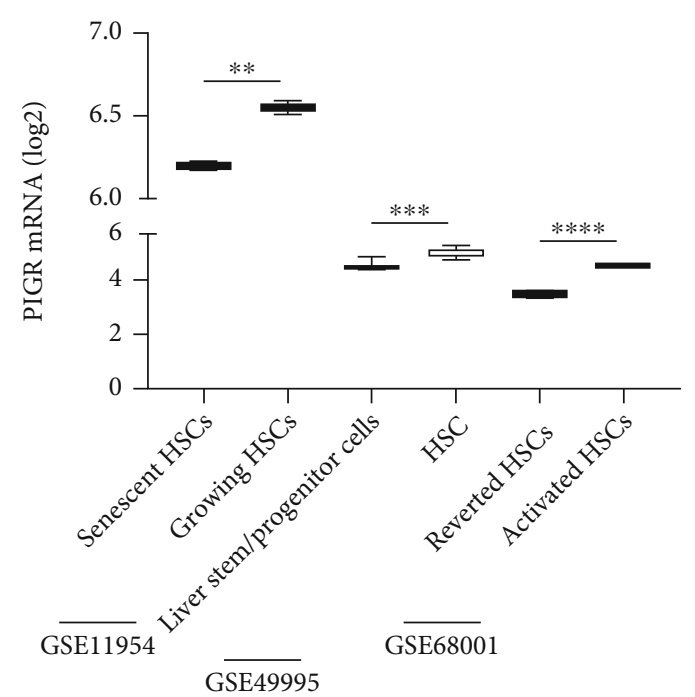

(c)

FIGURE 2: PIGR protein staining in liver tissues (a); PIGR mRNA expression in the single cell type clusters identified in liver tissue (b); and PIGR mRNA expression comparison in different status HSCs (c).

Metascape. As summarized in Figure 4, defense response to virus, interferon (IFN) signaling, and regulation of immune effector process were mainly enriched (Figures 4(a) and 4(b)). Additionally, KEGG, Reactome, and GO enrichment of similar/interacted genes of PIGR were reevaluated in GSEA. In line with results in Metascape, defense response to virus, response to virus, and response to type I IFN were mainly enriched in GO in GSEA. Antiviral response and
IFN alpha/beta signaling were mainly enriched in Reactome. Moreover, pathways involved in immune responses were enriched in KEGG in GSEA (Figure 5(a)).

In DisGeNET, neoplastic process including adenocarcinoma, squamous cell carcinoma, colon carcinoma, carcinoma of lung, tumor progression, pancreatic carcinoma, malignant neoplasm of lung, and small cell carcinoma was mainly associated with PIGR based on the current evidences 

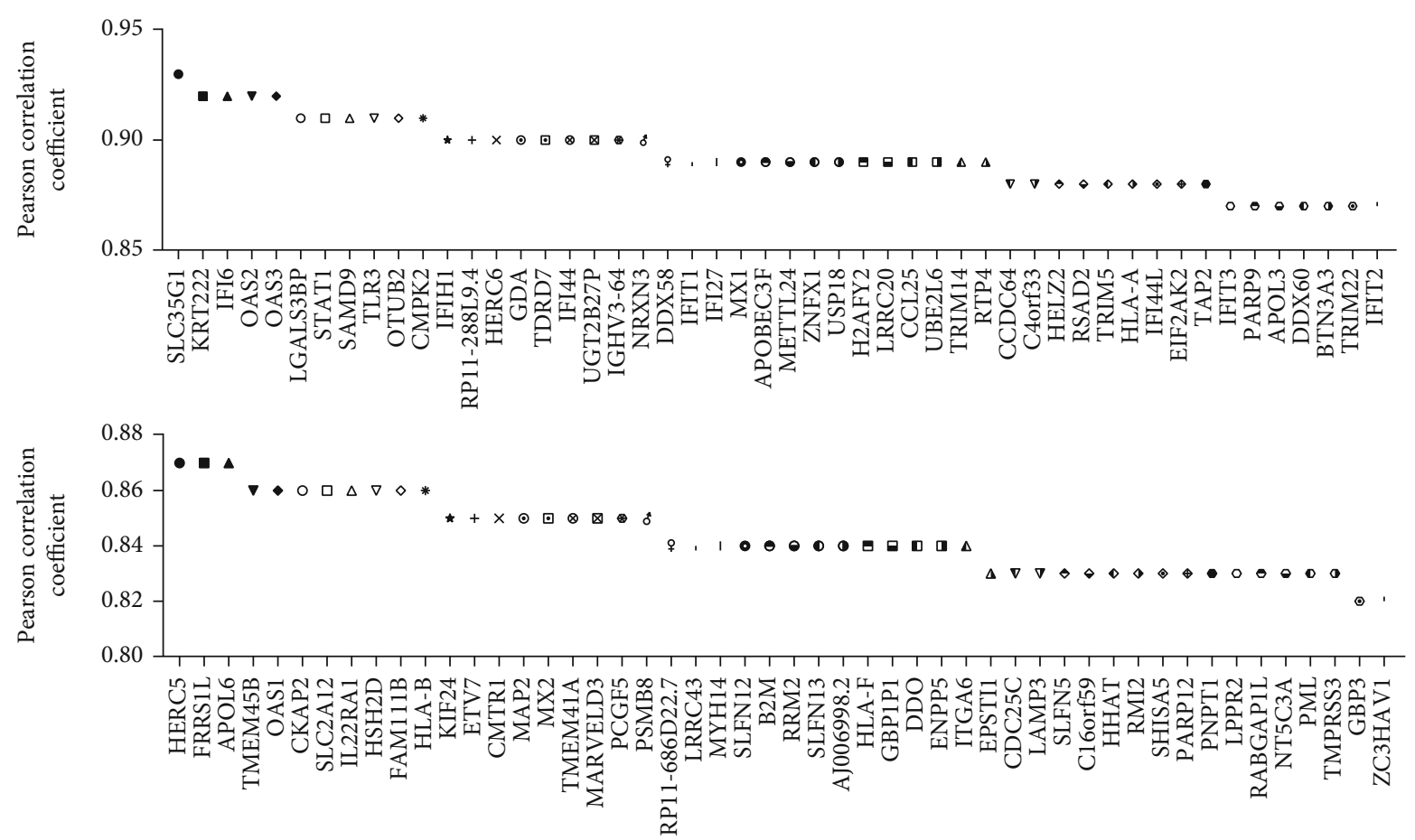

(a)

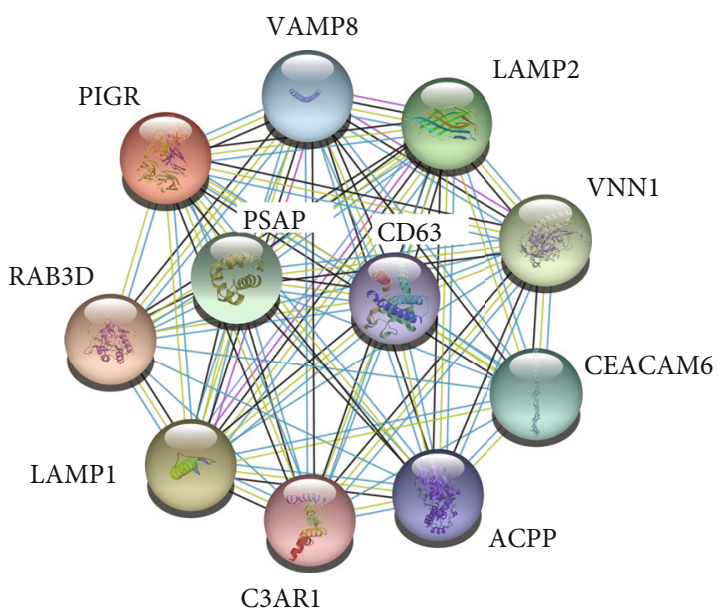

(b)

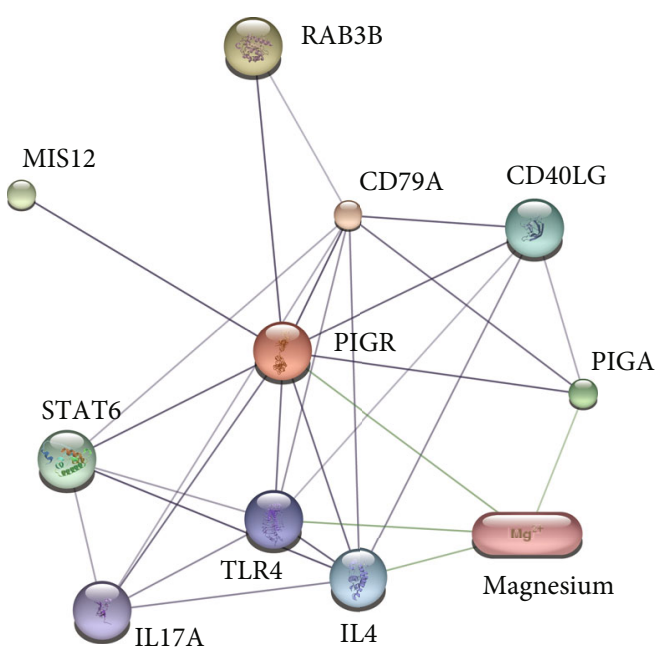

(c)

FIGURE 3: Top 100 similar genes of PIGR in GEPIA database (a) and protein-protein interaction (PPI) with PIGR in STRING (b) and STITCH (c).

from publications in PubMed (Figure 5(b)). As shown in Figure 5(b), no publications have investigated the correlations between PIGR and liver fibrosis.

3.4. PIGR Expression in Hepatitis Virus Infection. According to the enrichment analysis results, PIGR was mainly involved in virus- and IFN-related responses. Hence, we identified the PIGR expression levels in different hepatitis virus infections. In HBV infection, PIGR mRNA was significantly upregulated in $\mathrm{HBV}$-associated liver failure samples compared to normal individuals and liver angioma in GSE38941 [39] and GSE96851 [40] (both $p<0.001$, Figure 6(a)). Compared to HBV-negative samples, PIGR mRNA was significantly overexpressed in HBV-positive liver samples in GSE118295 $(p<0.01$, Figure 6(a)). In addition, PIGR mRNA was also significantly upregulated in PBMC samples from immune clearance $\mathrm{CHB}$ patients compared to that from inactive carrier and immune tolerance ones $(p<0.001$, Figure 6(a)). Intriguingly, PIGR mRNA was significantly higher in preIFN alpha-2b livers than that in post-IFN alpha-2b samples $(p<0.01$, Figure 6(a)) [41].

Compared to control chimpanzees, PIGR mRNA was significantly overexpressed in $\mathrm{HCV}$-infected ones in GSE22160 [42] $(p<0.01$, Figure 6(b)). Compared to control mice and control mice treated with IFN-alpha, PIGR mRNA was significantly upregulated in HCV-infected mice in GSE37715 (both $p<0.05$, Figure 6(b)). In addition, PIGR mRNA was significantly higher in chronic HCV- 

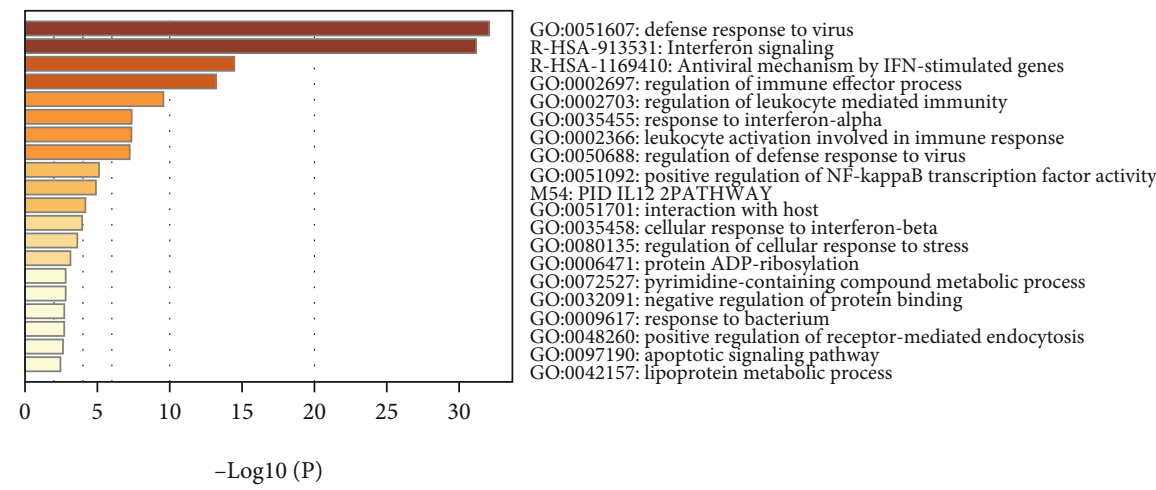

(a)

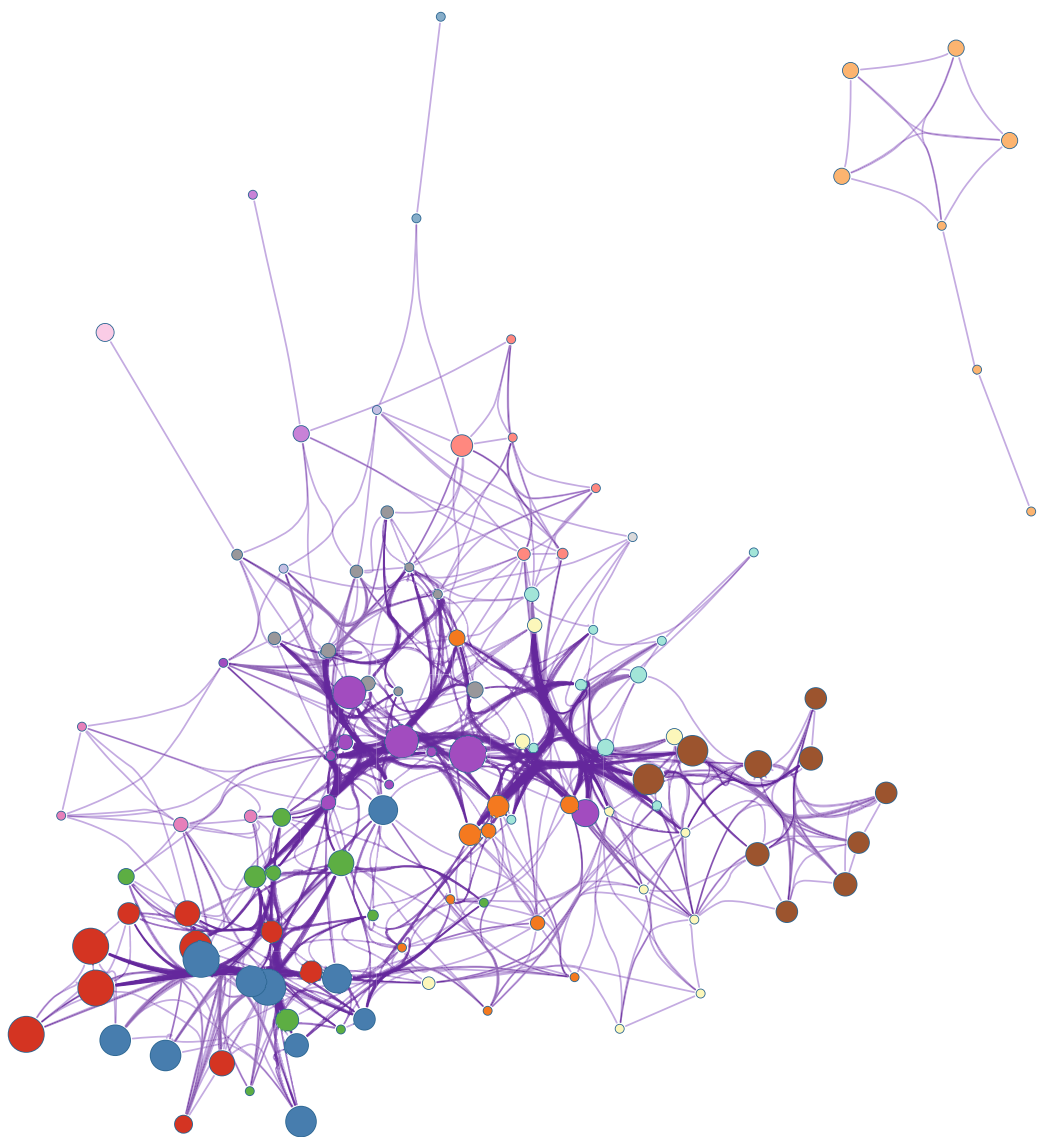

Defense response to virus

Interferon signaling

Antiviral mechanism by IFN-stimulated genes

Regulation of immune effector process

Regulation of leukocyte mediated immunity

Response to interferon-alpha

Leukocyte activation involved in immune response

Regulation of defense response to virus

Positive regulation of NF-kappaB transcription factor activity

PID IL12 2PATHWAY

Interaction with host

Cellular response to interferon-beta

Regulation of cellular response to stress

Protein ADP-ribosylation

Negative regulation of protein binding

- Pyrimidine-containing compound metabolic process

Response to bacterium

Positive regulation of receptor-mediated endocytosis

- Apoptotic signaling pathway

Lipoprotein metabolic process

(b)

FIGURE 4: Enrichment of similar/interacted genes of PIGR in Metascape database. 


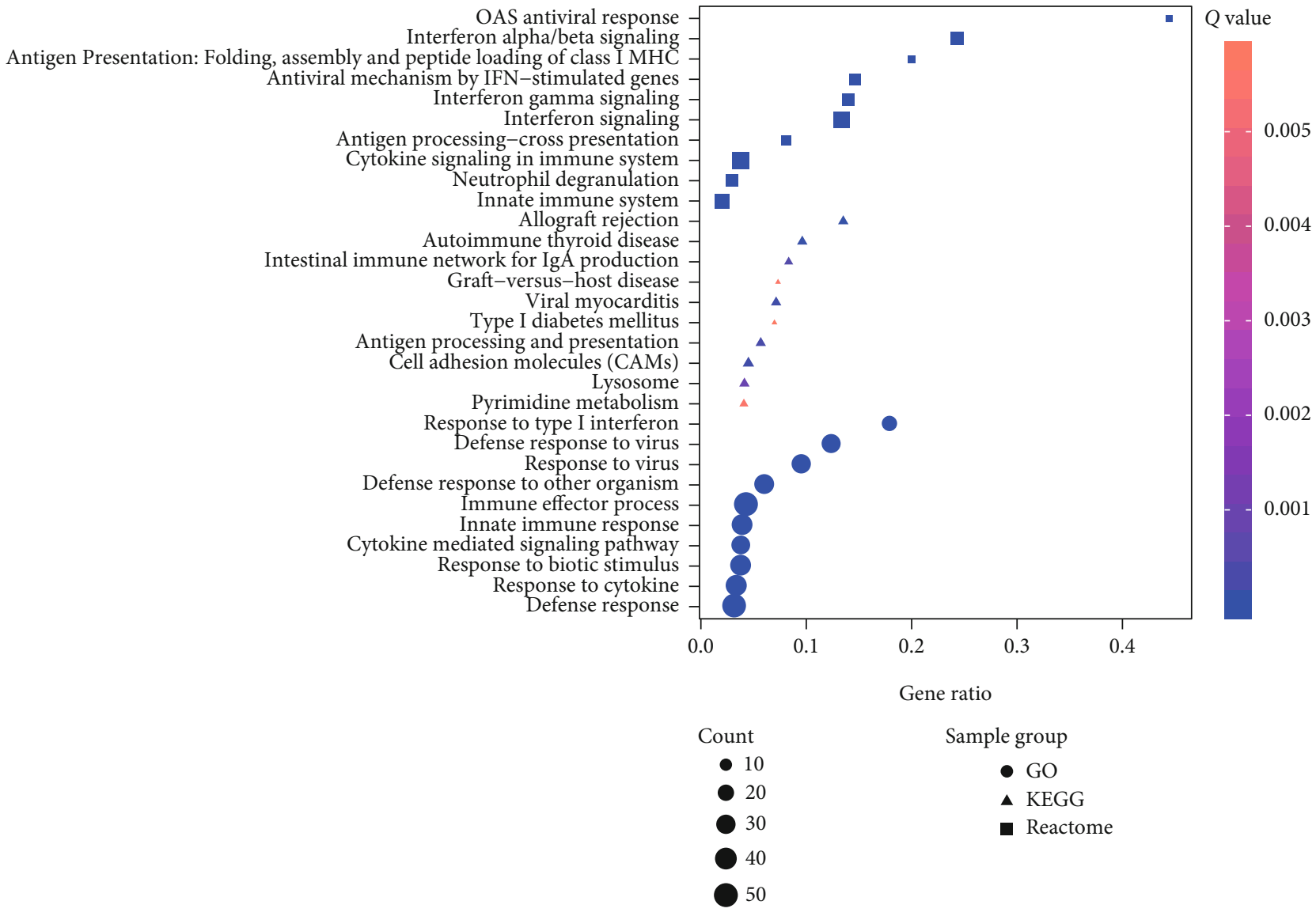

(a)

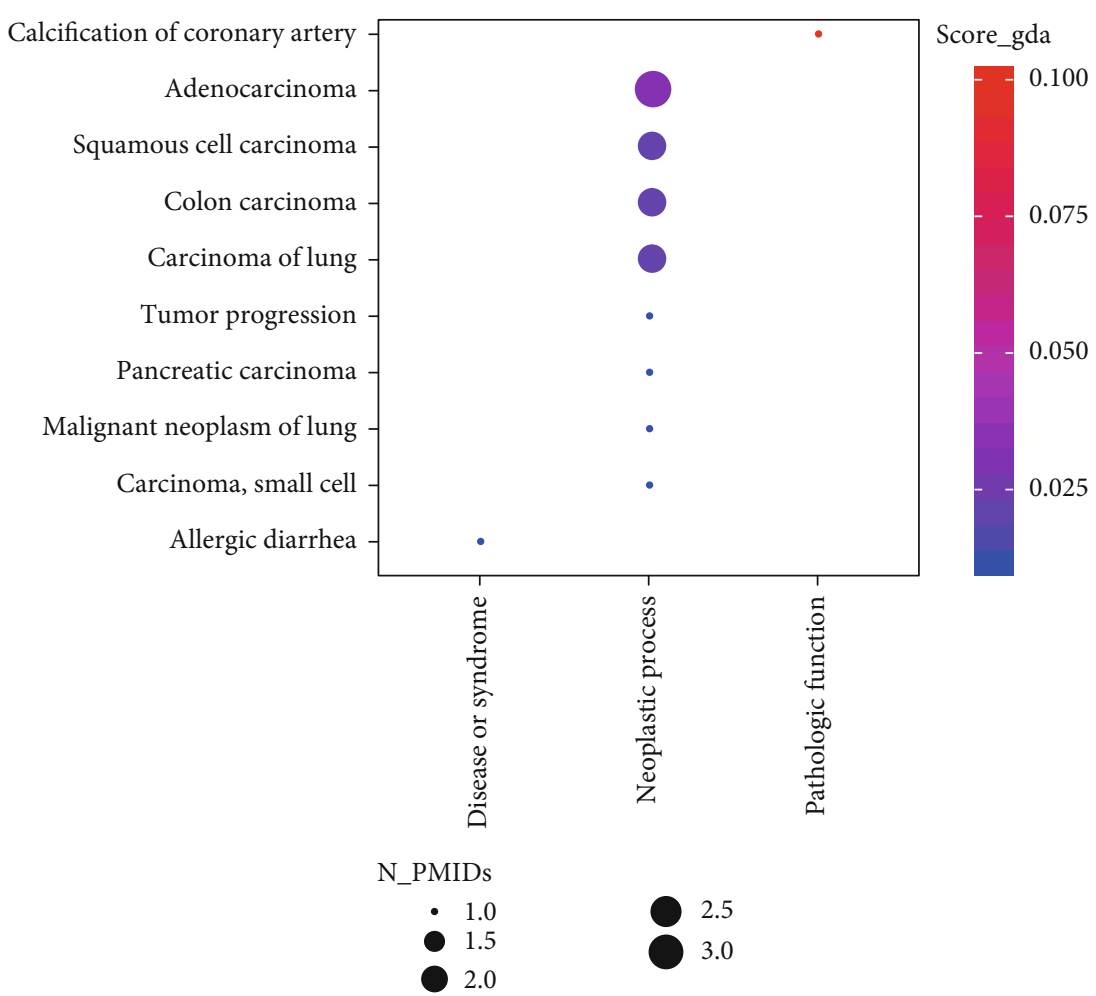

(b)

FIGURE 5: Enrichment of similar/interacted genes of PIGR in GSEA (a) and PIGR-related diseases in DisGeNET (b). 


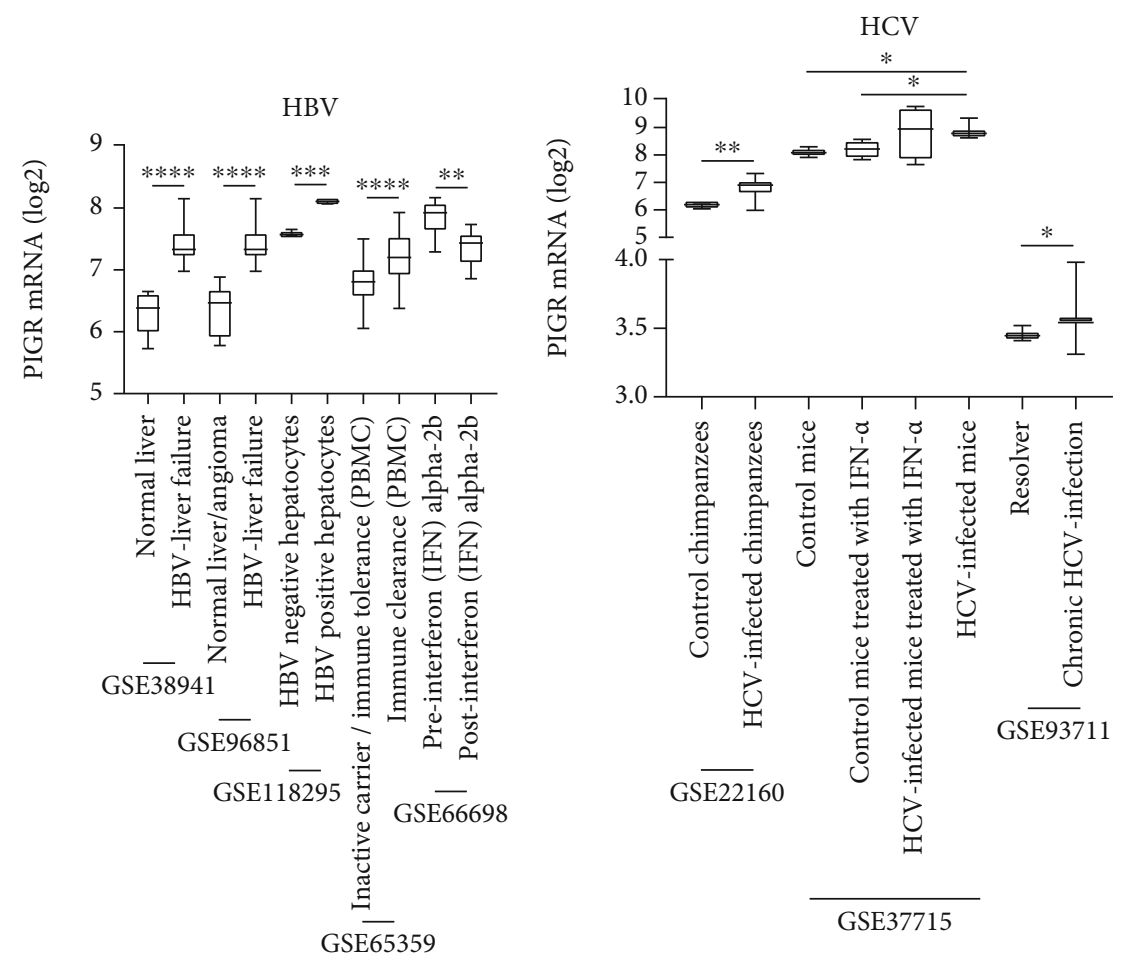

(a)

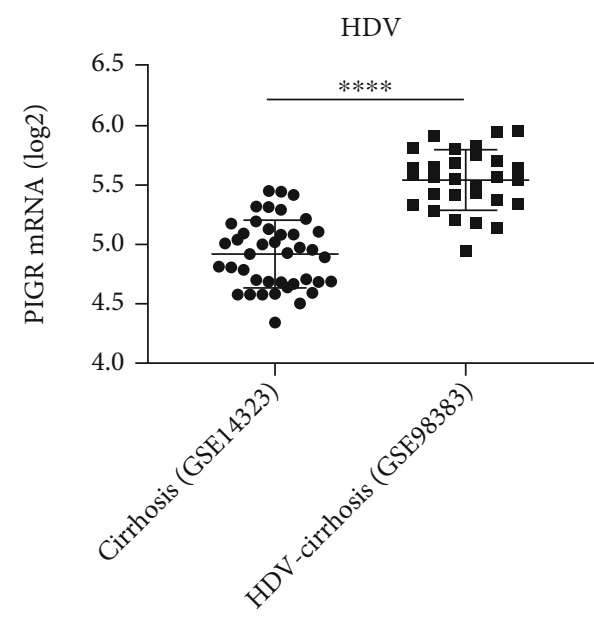

(c) (b)

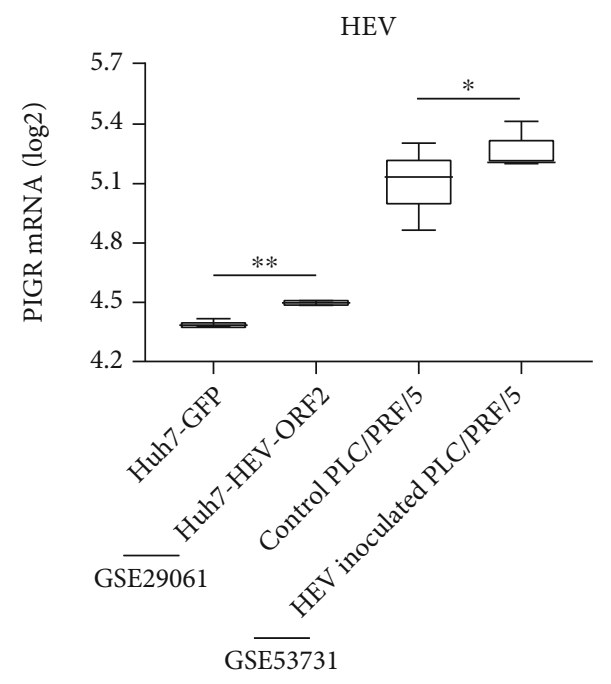

(d)

Figure 6: PIGR mRNA expression levels according to hepatitis virus B (a), C (b), D (c), and E (d) infection.

infection than that in resolver in GSE93711 [43] $(p<0.05$, Figure 6(b)).

PIGR mRNA was significantly upregulated in HDVrelated cirrhosis in GSE98383 [44] compared to cirrhosis samples from GSE14323 [34] ( $p<0.001$, Figure 6(c)).

PIGR mRNA was also significantly overexpressed in Huh7 cells with recombinant adenovirus encoding the HEV ORF2 compared to that in adenovirus encoding the green fluorescent protein in GSE29061 [45] ( $p<0.01$, Figure 6(d)). In addition, PIGR mRNA was significantly upregulated in $\mathrm{PLC} / \mathrm{PRF} / 5$ cells inoculated with HEV than that in PLC/PRF/5 cells inoculated with serum-free DMEM/199 medium in GSE53731 [46] $(p<0.05$, Figure 6(d)).

3.5. Associations between pIgR and Fibrosis Stage in $\mathrm{CHB}$ Patients. In GSE84044 [35], information including liver fibrosis stage, age, and gender of $124 \mathrm{CHB}$ patients were obtained from GEO database. After adjusting age and gender, multivariate logistic regression models revealed that high PIGR in liver was a risk factor for liver fibrosis $(\mathrm{OR}=82.2$, $95 \% \mathrm{CI}=14.4-469.4, p<0.001$, Figure $7(\mathrm{a}))$. The optimal cutoff of PIGR for predicting liver fibrosis stage $\geq 2$ was 


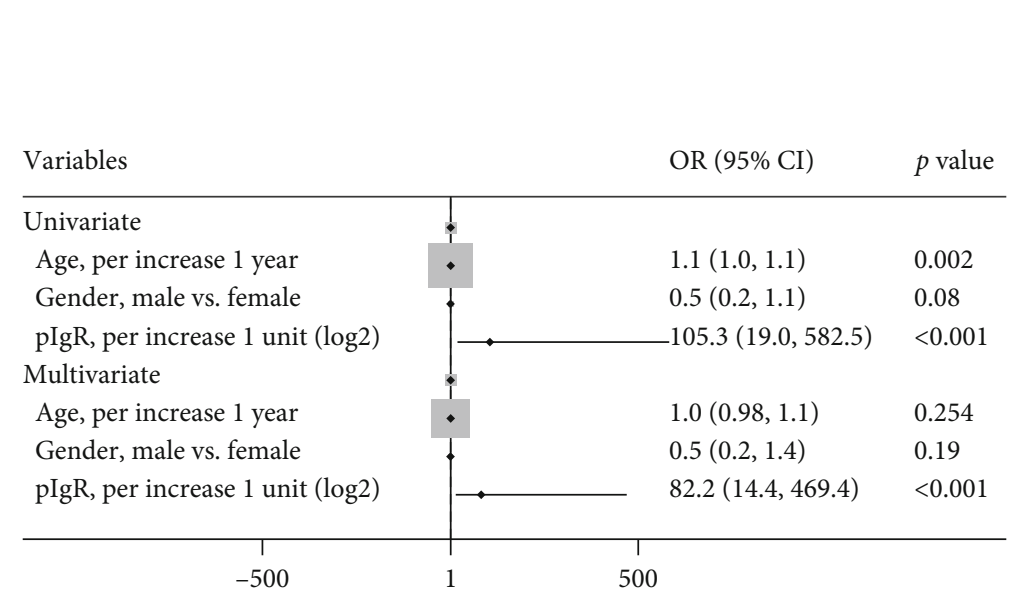

(a)

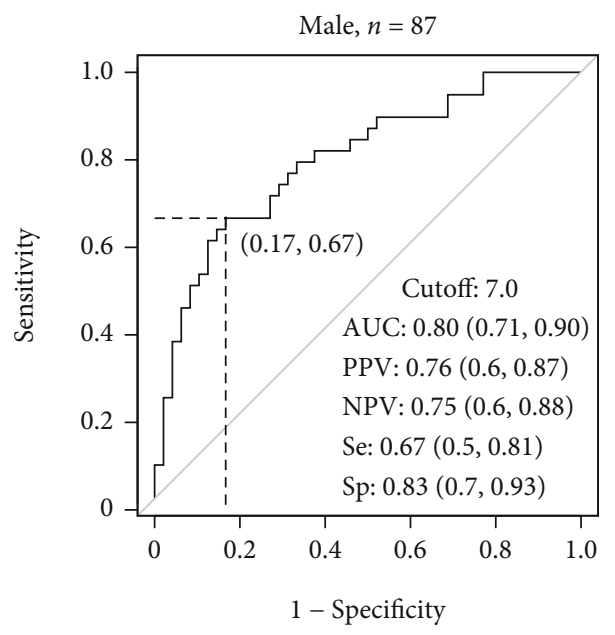

(c)

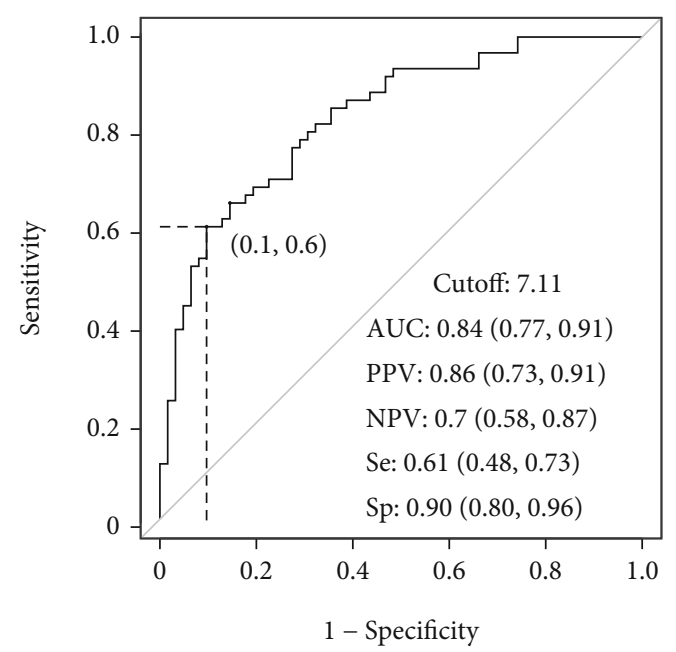

(b)

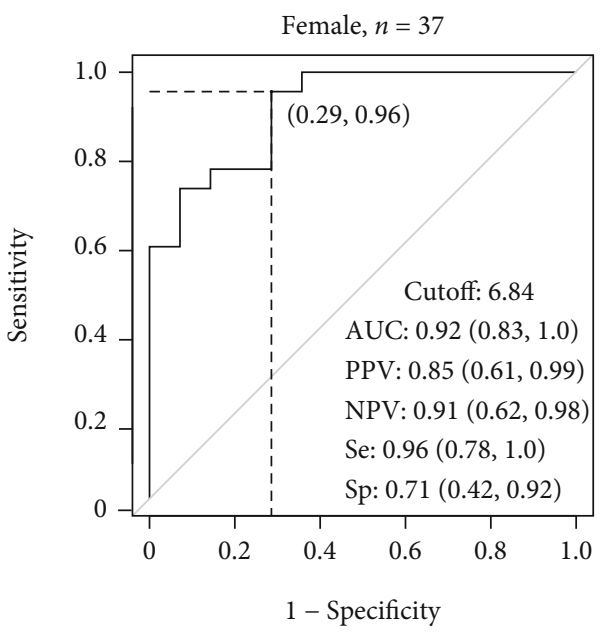

(d)

FIGURE 7: Logistic regression analysis of parameters associated with liver fibrosis stage $\geq 2$ in chronic hepatitis B patients (a) and ROC curves of PIGR for liver fibrosis stage $\geq 2$ in CHB patients (b-d) in GSE84044.

7.11. The area under curve (AUC), positive predictive value (PPV), negative predictive value (NPV), sensitivity, and specificity were $0.84,0.86,0.70,0.61$, and 0.90 , respectively (Figure 7(b)).

We conducted subgroup analysis by gender. In male population, the optimal cutoff of PIGR for predicting liver fibrosis stage $\geq 2$ was 7.0. The AUC, PPV, NPV, sensitivity, and specificity were $0.80,0.76,0.75,0.67$, and 0.83 , respectively (Figure $7(\mathrm{c})$ ). In female, the optimal cutoff of PIGR for predicting liver fibrosis stage $\geq 2$ was 6.84. The AUC, PPV, NPV, sensitivity, and specificity were $0.92,0.85,0.91$, 0.96 , and 0.71 , respectively (Figure $7(\mathrm{~d})$ ).

\section{Discussion}

PIGR was generally known as a mediator of transcytosis of polymeric immunoglobulins, accelerating the secretion of IgA and IgM and comprising the defense line against infection [1]. Recently, Nallagangula et al. and Niu et al. revealed the associations between PIGR and liver cirrhosis
$[16,17]$. In addition, Ai et al., Yue et al., and our previous studies have uncovered the unrecognized roles of PIGR in the promotion of tumorigenesis and metastases [12, 14, $15,47]$. On the other side, they identified the potential inflammation links between $\mathrm{HBV}$ infection and cancer malignancy $[12,14]$. Considering the underlying correlations between hepatitis virus infection, liver fibrosis, and hepatocarcinogenesis, we assumed that PIGR might participated in the liver fibrosis progression, which resulted in liver malignancies.

In this analysis, we found that PIGR was apparently upregulated in advanced liver fibrosis. And high PIGR expression could reliably predict the liver fibrosis stage. Our previous publication revealed that PIGR was overexpressed in tumor tissues compared to adjacent tissues in hepatocellular carcinoma [47]. Thus, PIGR might promote the processes of liver fibrosis, cirrhosis, and hepatocarcinogenesis in the chronic liver diseases. Multiple intracellular signaling pathways including Janus kinase-signal transduction and activator of transcription (JAK-STAT), NF- $\kappa \mathrm{B}$, 
and mitogen-activated protein kinase (MAPK) are involved in the regulation of PIGR [1-3]. Abnormal cytokine cross talk between epithelium and inflammatory cells contributed to fibrosis [48]. And the JAK-STAT, NF- $\kappa$ B, and MAPK pathways were the main signaling in regulating liver fibrosis and regeneration induced by these cytokines [49-51]. Given the recently established connections between PIGR, proinflammatory cytokines, and signaling pathways including JAK-STAT, NF- $\kappa \mathrm{B}$, and MAPK, our findings partly raise the underlying possibility that PIGR may facilitate liver fibrosis via proinflammatory cytokineinduced pathways.

HSCs and hepatitis virus infection are vital factors in liver fibrosis progression. Our results revealed that PIGR expression is in line with the activation of HSCs and the status of hepatitis virus infection. HSCs play a key role in the initiation, progression, and regression of liver fibrosis [52, 53], and activation of HSCs is the major cellular driver of liver fibrogenesis [54]. Proinflammatory cytokines, which could simulate the expression of PIGR, could promote transdifferentiation of quiescent HSCs into activated HSCs in fibrogenic liver and facilitate secretion of extracellular matrix molecules $[52,54]$. On the other side, chronic hepatitis virus infection is one of the major risk factors for fibrotic liver diseases. Existed evidence showed that HBV e antigen, core antigen, and X proteins could directly induce activation and proliferation of HSCs [54, 55]. And HCV viral core and nonstructural proteins directly induce inflammatory and profibrogenic pathways including Ras/ERK, PI3K/AKT, and TGF- $\beta$ signaling in HSCs [56]. Clinical studies also indicated that serum IgA level is positively correlated with the severity of liver fibrosis and functions as in independent predictor for cirrhosis [57, 58]. Thus, we drew the hypothesis that PIGR might be involved in the fibrosis progression via hepatitis virus- and HSC-related mechanisms.

This analysis has some limitations. Firstly, we conducted a bioinformatic analysis, without experimental validation of our results. Secondly, limited parameters were included in the logistic model; confounding variables might exist. Thirdly, PIGR expression between normal and cirrhotic samples is still controversial in multiple public datasets. Fourthly, PIGR was mainly detected in cholangiocytes and hepatocytes, not in HSCs, in HPA database. Hence, the causality between PIGR and liver fibrosis needs further confirmation, even though our results provided the potential correlations between PIGR, HSCs, hepatitis virus infection, and liver fibrosis progression, which might be helpful for identification of novel therapeutic targets of regression of liver fibrosis.

\section{Data Availability}

Datasets of the current study are available from the NCBI Gene Expression Omnibus (https://www.ncbi.nlm.nih.gov/ geo/), Oncomine (https://www.oncomine.org/), and the Human Protein Atlas (HPA, https://www.proteinatlas.org/) databases. All the datasets were available from the corresponding authors with reasonable request.

\section{Disclosure}

The funders had no role in study design, data collection and analysis, decision to publish, or preparation of the manuscript.

\section{Conflicts of Interest}

The authors declared no conflicts of interest in this work.

\section{Authors' Contributions}

Yuan Zhang and Wenjun Lu contributed equally to this work.

\section{Acknowledgments}

This work was supported by the National Natural Science Foundation of China (81803901).

\section{Supplementary Materials}

Supplementary Table S1: the details of GEO series included in this analysis. (Supplementary Materials)

\section{References}

[1] M. Asano and K. Komiyama, "Polymeric immunoglobulin receptor," Journal of Oral Science, vol. 53, no. 2, pp. 147-156, 2011.

[2] F. E. Johansen and C. S. Kaetzel, "Regulation of the polymeric immunoglobulin receptor and IgA transport: new advances in environmental factors that stimulate pIgR expression and its role in mucosal immunity," Mucosal Immunology, vol. 4, no. 6, pp. 598-602, 2011.

[3] C. S. Kaetzel, "Cooperativity among secretory IgA, the polymeric immunoglobulin receptor, and the gut microbiota promotes host-microbial mutualism," Immunology Letters, vol. 162, no. 2, pp. 10-21, 2014.

[4] M. Hayashi, N. Takenouchi, M. Asano et al., "The polymeric immunoglobulin receptor (secretory component) in a human intestinal epithelial cell line is up-regulated by interleukin-1," Immunology, vol. 92, no. 2, pp. 220-225, 1997.

[5] S. Loman, H. M. Jansen, T. A. Out, and R. Lutter, "Interleukin4 and interferon-gamma synergistically increase secretory component gene expression, but are additive in stimulating secretory immunoglobulin A release by Calu-3 airway epithelial cells," Immunology, vol. 96, no. 4, pp. 537-543, 1999.

[6] Y. Nihei, K. Maruyama, Y. Endo, T. Sato, K. Kobayashi, and F. Kaneko, "Secretory component (polymeric immunoglobulin receptor) expression on human keratinocytes by stimulation with interferon- $\gamma$ and differences in response," Journal of Dermatological Science, vol. 11, no. 3, pp. 214-222, 1996.

[7] L. M. Sollid, D. Kvale, P. Brandtzaeg, G. Markussen, and E. Thorsby, "Interferon-gamma enhances expression of secretory component, the epithelial receptor for polymeric immunoglobulins," Journal of Immunology, vol. 138, no. 12, pp. 4303-4306, 1987.

[8] J. O. Phillips, M. P. Everson, Z. Moldoveanu, C. Lue, and J. Mestecky, "Synergistic effect of IL-4 and IFN-gamma on the expression of polymeric Ig receptor (secretory component) and IgA binding by human epithelial cells," Journal of Immunology, vol. 145, no. 6, pp. 1740-1744, 1990. 
[9] P. Arumugam, S. Bhattacharya, J. Chin-Aleong, M. Capasso, and H. M. Kocher, "Expression of polymeric immunoglobulin receptor and stromal activity in pancreatic ductal adenocarcinoma," Pancreatology, vol. 17, no. 2, pp. 295-302, 2017.

[10] M. Bomsel, M. Heyman, H. Hocini et al., "Intracellular neutralization of HIV transcytosis across tight epithelial barriers by anti-HIV envelope protein $\operatorname{dIgA}$ or IgM," Immunity, vol. 9, no. 2, pp. 277-287, 1998.

[11] H. Yan, M. E. Lamm, E. Bjorling, and Y. T. Huang, "Multiple functions of immunoglobulin $\mathrm{A}$ in mucosal defense against viruses: an in vitro measles virus model," Journal of Virology, vol. 76, no. 21, pp. 10972-10979, 2002.

[12] J. Ai, Q. Tang, Y. Wu et al., "The role of polymeric immunoglobulin receptor in inflammation-induced tumor metastasis of human hepatocellular carcinoma," Journal of the National Cancer Institute, vol. 103, no. 22, pp. 1696-1712, 2011.

[13] N. Sphyris and S. A. Mani, "pIgR: frenemy of inflammation, EMT, and HCC progression," Journal of the National Cancer Institute, vol. 103, no. 22, pp. 1644-1645, 2011.

[14] X. Yue, J. Ai, Y. Xu et al., "Polymeric immunoglobulin receptor promotes tumor growth in hepatocellular carcinoma," Hepatology, vol. 65, no. 6, pp. 1948-1962, 2017.

[15] Y. Zhang, J. Zhang, X. Chen, and Z. Yang, "Polymeric immunoglobulin receptor (PIGR) exerts oncogenic functions via activating ribosome pathway in hepatocellular carcinoma," International Journal of Medical Sciences, vol. 18, no. 2, pp. 364-371, 2021.

[16] K. S. Nallagangula, V. Lakshmaiah, C. Muninarayana, K. V. Deepa, and K. N. Shashidhar, "A proteomic approach of biomarker candidate discovery for alcoholic liver cirrhosis," Journal of Circulating Biomarkers, vol. 7, p. 184945441878841, 2018.

[17] L. Niu, P. E. Geyer, N. J. Wewer Albrechtsen et al., "Plasma proteome profiling discovers novel proteins associated with non-alcoholic fatty liver disease," Molecular Systems Biology, vol. 15, no. 3, p. e8793, 2019.

[18] T. Barrett, S. E. Wilhite, P. Ledoux et al., "NCBI GEO: archive for functional genomics data sets-update," Nucleic Acids Research, vol. 41, no. Database issue, pp. D991-D995, 2013.

[19] S. E. Wilhite and T. Barrett, "Strategies to explore functional genomics data sets in NCBI's GEO database," Methods in Molecular Biology, vol. 802, pp. 41-53, 2012.

[20] L. Gautier, L. Cope, B. M. Bolstad, and R. A. Irizarry, "affyanalysis of Affymetrix GeneChip data at the probe level," Bioinformatics, vol. 20, no. 3, pp. 307-315, 2004.

[21] M. E. Ritchie, B. Phipson, D. Wu et al., "limma powers differential expression analyses for RNA-sequencing and microarray studies," Nucleic Acids Research, vol. 43, no. 7, article e47, 2015.

[22] D. R. Rhodes, J. Yu, K. Shanker et al., "ONCOMINE: a cancer microarray database and integrated data-mining platform," Neoplasia, vol. 6, no. 1, pp. 1-6, 2004.

[23] D. R. Rhodes, S. Kalyana-Sundaram, V. Mahavisno et al., "Oncomine 3.0: genes, pathways, and networks in a collection of 18,000 cancer gene expression profiles," Neoplasia, vol. 9, no. 2, pp. 166-180, 2007.

[24] M. Uhlen, L. Fagerberg, B. M. Hallstrom et al., "Proteomics. Tissue-based map of the human proteome," Science, vol. 347, no. 6220, p. 1260419, 2015.

[25] J. Pinero, A. Bravo, N. Queralt-Rosinach et al., "DisGeNET: a comprehensive platform integrating information on human disease-associated genes and variants," Nucleic Acids Research, vol. 45, no. D1, pp. D833-D839, 2017.

[26] J. Pinero, N. Queralt-Rosinach, A. Bravo et al., "DisGeNET: a discovery platform for the dynamical exploration of human diseases and their genes," Database (Oxford), vol. 2015, 2015.

[27] J. Pinero, J. M. Ramirez-Anguita, J. Sauch-Pitarch et al., "The DisGeNET knowledge platform for disease genomics: 2019 update," Nucleic Acids Research, vol. 48, no. D1, pp. D845D855, 2020.

[28] Z. Tang, C. Li, B. Kang, G. Gao, C. Li, and Z. Zhang, "GEPIA: a web server for cancer and normal gene expression profiling and interactive analyses," Nucleic Acids Research, vol. 45, no. W1, pp. W98-W102, 2017.

[29] Y. Zhou, B. Zhou, L. Pache et al., "Metascape provides a biologist-oriented resource for the analysis of systems-level datasets," Nature Communications, vol. 10, no. 1, p. 1523, 2019.

[30] V. K. Mootha, C. M. Lindgren, K.-F. Eriksson et al., "PGC-1 $\alpha$ responsive genes involved in oxidative phosphorylation are coordinately downregulated in human diabetes," Nature Genetics, vol. 34, no. 3, pp. 267-273, 2003.

[31] A. Subramanian, P. Tamayo, V. K. Mootha et al., "Gene set enrichment analysis: a knowledge-based approach for interpreting genome-wide expression profiles," Proceedings of the National Academy of Sciences of the United States of America, vol. 102, no. 43, pp. 15545-15550, 2005.

[32] M. Lopez-Raton, M. X. Rodriguez-Alvarez, C. C. Suarez, and F. G. Sampedro, "OptimalCutpoints: AnRPackage for selecting optimal cutpoints in diagnostic tests," Journal of Statistical Software, vol. 61, no. 8, 2014.

[33] E. Wurmbach, Y. B. Chen, G. Khitrov et al., "Genome-wide molecular profiles of HCV-induced dysplasia and hepatocellular carcinoma," Hepatology, vol. 45, no. 4, pp. 938-947, 2007.

[34] V. R. Mas, D. G. Maluf, K. J. Archer et al., "Genes involved in viral carcinogenesis and tumor initiation in hepatitis $\mathrm{C}$ virusinduced hepatocellular carcinoma," Molecular Medicine, vol. 15, no. 3-4, pp. 85-94, 2009.

[35] M. Wang, Q. Gong, J. Zhang et al., "Characterization of gene expression profiles in HBV-related liver fibrosis patients and identification of ITGBL1 as a key regulator of fibrogenesis," Scientific Reports, vol. 7, no. 1, 2017.

[36] V. Krizhanovsky, M. Yon, R. A. Dickins et al., "Senescence of activated stellate cells limits liver fibrosis," Cell, vol. 134, no. 4, pp. 657-667, 2008.

[37] S. Berardis, C. Lombard, J. Evraerts et al., "Gene expression profiling and secretome analysis differentiate adult-derived human liver stem/progenitor cells and human hepatic stellate cells," PLoS One, vol. 9, no. 1, article e86137, 2014.

[38] A. El Taghdouini, M. Najimi, P. Sancho-Bru, E. Sokal, and L. A. van Grunsven, "In vitro reversion of activated primary human hepatic stellate cells," Fibrogenesis \& Tissue Repair, vol. 8, no. 1, 2015.

[39] O. Nissim, M. Melis, G. Diaz et al., "Liver regeneration signature in hepatitis B virus (HBV)-associated acute liver failure identified by gene expression profiling," PLoS One, vol. 7, no. 11, article e49611, 2012.

[40] Z. Chen, G. Diaz, T. Pollicino et al., "Role of humoral immunity against hepatitis $\mathrm{B}$ virus core antigen in the pathogenesis of acute liver failure," Proceedings of the National Academy of Sciences of the United States of America, vol. 115, no. 48, pp. E11369-E11378, 2018. 
[41] H.-L. Wu, T.-H. Hsiao, P.-J. Chen et al., "Liver gene expression profiles correlate with virus infection and response to interferon therapy in chronic hepatitis B patients," Scientific Reports, vol. 6, 2016.

[42] C. Yu, D. Boon, S. L. McDonald et al., "Pathogenesis of hepatitis $\mathrm{E}$ virus and hepatitis $\mathrm{C}$ virus in chimpanzees: similarities and differences," Journal of Virology, vol. 84, no. 21, pp. 11264-11278, 2010.

[43] D. Wolski, P. K. Foote, D. Y. Chen et al., "Early transcriptional divergence marks virus-specific primary human $\mathrm{CD}^{+} \mathrm{T}$ cells in chronic versus acute infection," Immunity, vol. 47, no. 4, pp. 648-663.e8, 2017, e648.

[44] G. Diaz, R. E. Engle, A. Tice et al., "Molecular signature and mechanisms of hepatitis D virus-associated hepatocellular carcinoma," Molecular Cancer Research, vol. 16, no. 9, pp. 14061419, 2018.

[45] L. John, S. Thomas, O. Herchenroder, B. M. Putzer, and S. Schaefer, "Hepatitis E virus ORF2 protein activates the pro-apoptotic gene CHOP and anti-apoptotic heat shock proteins," PLoS One, vol. 6, no. 9, article e25378, 2011.

[46] F. Zhang, Y. Qi, T. J. Harrison et al., "Hepatitis E genotype 4 virus from feces of monkeys infected experimentally can be cultured in PLC/PRF/5 cells and upregulate host interferoninducible genes," Journal of Medical Virology, vol. 86, no. 10, pp. 1736-1744, 2014.

[47] Z. Yang, Y. Tao, X. Xu, F. Cai, Y. Yu, and L. Ma, "Bufalin inhibits cell proliferation and migration of hepatocellular carcinoma cells via APOBEC3F induced intestinal immune network for IgA production signaling pathway," Biochemical and Biophysical Research Communications, vol. 503, no. 3, pp. 2124-2131, 2018.

[48] C. J. Song, K. A. Zimmerman, S. J. Henke, and B. K. Yoder, "Inflammation and fibrosis in polycystic kidney disease," Results and Problems in Cell Differentiation, vol. 60, pp. 323344, 2017.

[49] X. Kong, N. Horiguchi, M. Mori, and B. Gao, "Cytokines and STATs in liver fibrosis," Frontiers in Physiology, vol. 3, 2012.

[50] N. Huang, F. Ji, S. Zhang et al., "Effect of splenectomy on serum cytokine profiles in hepatitis B virus-related cirrhosis patients with portal hypertension," Viral Immunology, vol. 31, no. 5, pp. 371-378, 2018.

[51] H. H. Kang, I. K. Kim, H. I. Lee et al., "Chronic intermittent hypoxia induces liver fibrosis in mice with diet-induced obesity via TLR4/MyD88/MAPK/NF-kB signaling pathways," Biochemical and Biophysical Research Communications, vol. 490, no. 2, pp. 349-355, 2017.

[52] C. Y. Zhang, W. G. Yuan, P. He, J. H. Lei, and C. X. Wang, "Liver fibrosis and hepatic stellate cells: etiology, pathological hallmarks and therapeutic targets," World Journal of Gastroenterology, vol. 22, no. 48, pp. 10512-10522, 2016.

[53] J. E. Puche, Y. Saiman, and S. L. Friedman, "Hepatic stellate cells and liver fibrosis," Comprehensive Physiology, vol. 3, no. 4, pp. 1473-1492, 2013.

[54] T. Higashi, S. L. Friedman, and Y. Hoshida, "Hepatic stellate cells as key target in liver fibrosis," Advanced Drug Delivery Reviews, vol. 121, pp. 27-42, 2017.

[55] Q. Bai, J. An, X. Wu et al., "HBV promotes the proliferation of hepatic stellate cells via the PDGF-B/PDGFR- $\beta$ signaling pathway in vitro," International Journal of Molecular Medicine, vol. 30, no. 6, pp. 1443-1450, 2012.
[56] R. Bataller, Y. H. Paik, J. N. Lindquist, J. J. Lemasters, and D. A. Brenner, "Hepatitis C virus core and nonstructural proteins induce fibrogenic effects in hepatic stellate cells," Gastroenterology, vol. 126, no. 2, pp. 529-540, 2004.

[57] S. Lin, Q. Sun, W. Mao, and Y. Chen, "Serum immunoglobulin A (IgA) level is a potential biomarker indicating cirrhosis during chronic hepatitis B infection," Gastroenterology Research and Practice, vol. 2016, Article ID 2495073, 2016.

[58] I. Maleki, M. R. Aminafshari, T. Taghvaei et al., "Serum immunoglobulin A concentration is a reliable biomarker for liver fibrosis in non-alcoholic fatty liver disease," World Journal of Gastroenterology, vol. 20, no. 35, pp. 12566-12573, 2014. 